\title{
A DECOMPOSITION OF MIXED ABELIAN GROUPS
}

\author{
BY \\ JOHN A. OPPELT $\left.{ }^{(}\right)$
}

1. Introduction. For each torsion group $T$ and torsion-free group $J$, what conditions must be satisfied by $T$ and $J$ so that every extension $G$ of $T$ by $J$ is a direct sum of groups whose torsion subgroups are primary? Adapting the methods used by Baer in [1] we obtain, in the case that $J$ is a completely decomposable group, necessary and sufficient conditions which depend only on the divisibility properties of $T$ and $J$ (see Theorem 2.4).

We will name a mixed group, whose maximal torsion subgroup is $p$-primary, a $p$-mixed group. $\operatorname{Ext}(J, T)$ consists of equivalence classes of extensions of the form

$$
E: 0 \rightarrow T \rightarrow X \rightarrow J \rightarrow 0 .
$$

By $G \in \in \operatorname{Ext}(J, T)$ we mean that some extension of the form $E$ with the group $G$ as the middle term belongs to an equivalence class in $\operatorname{Ext}(J, T)$. Finally $[G]$ denotes an equivalence class of $G \in \in \operatorname{Ext}(J, T)$.

2. The main theorems. We begin by establishing a sufficient condition for every $G \in \in \operatorname{Ext}(J, T)$ to be a direct sum of $p$-mixed groups. Here $J$ is torsion-free and $T$ is a torsion group. So the maximal torsion subgroup of $G$, which we call $t G$, is isomorphic to $T$ and $G / t G \cong J$. We designate the primary decomposition of $T$ by $T=\sum T_{p}$ where the sum is taken over relevant primes. $T^{q}$ will be the direct sum of all the primary components of $T$ except for $T_{q}$. Also note that $\operatorname{Ext}(J, T)=0$ means that every extension $G$ of $T$ by $J$ splits, i.e., $G \cong T \oplus J$.

THEOREM 2.1. Let $T=\sum T_{p}$ be a torsion group and $J$ be torsion-free. Suppose $J=\sum J_{p}$ such that $\operatorname{Ext}\left(J_{p}, T^{p}\right)=0$. Then every $G \in \in \operatorname{Ext}(J, T)$ is a direct sum of p-mixed groups.

Proof. We carry out the proof by computing in our setting, the standard isomorphisms

$$
\operatorname{Ext}(J, T) \cong \prod_{p} \operatorname{Ext}\left(J_{p}, T\right) \cong \prod_{p} \operatorname{Ext}\left(J_{p}, T_{p}\right)
$$

where the second isomorphism follows from $\operatorname{Ext}\left(J_{p}, T\right) \cong \operatorname{Ext}\left(J_{p}, T_{p}\right) \oplus \operatorname{Ext}\left(J_{p}, T^{p}\right)$, the last term being zero by hypothesis.

Received by the editors February 25, 1966.

(1) This work is contained in the author's doctoral dissertation and was partially supported by the National Science Foundation Grant GP-4. The author gratefully acknowledges the guidance of his director, George Kolettis, Jr. 
Let (4) $)_{p}$ below be any element of $\operatorname{Ext}\left(J_{p}, T_{p}\right)$ and form the element (2) of $\operatorname{Ext}(J, T)$, where $f$ is the Cartesian product of the maps $f_{p}$.

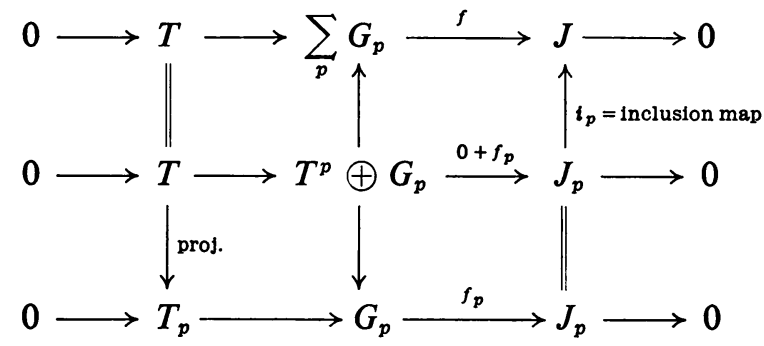

Commutativity of the diagram containing (2) and (3) $)_{p}$ shows that under the first isomorphism, call it $i^{*}$, of (1) the $p$ th coordinate of $i^{*}(2)$ is (3) $)_{p}$. Commutativity of the diagram containing $(3)_{p}$ and $(4)_{p}$ then shows that the image of $(3)_{p}$ under the second isomorphism of (1) is (4) $p$.

Since the extensions in (4) ${ }_{p}$ were arbitrary, we conclude that every element of $\operatorname{Ext}(J, T)$ has the form (2), as desired.

Remark. The author is indebted to the referee for the above less complicated proof and for an improved presentation of $\S 3$.

We now turn our attention to completely decomposable torsion-free groups. Let $J=\sum J_{\alpha}, \alpha \in I$, where $r\left(J_{\alpha}\right)=1$. Each $J_{\alpha}$ is characterized by its type (cf. [2, p. 207]), $\tau\left(J_{\alpha}\right)=\left(k_{1}^{\alpha}, k_{2}^{\alpha}, \ldots\right)$ where $k_{i}^{\alpha}$ is a nonnegative integer or $\infty$. For any group $G, p^{\omega} G$ is the subgroup of elements of infinite $p$-height. If $J$ is torsion-free of rank one then necessarily $p^{\omega} J=0$ or $p^{\omega} J=J$. If $J$ is completely decomposable, say $J=\sum J_{\alpha}, \alpha \in I$, then $p^{\omega} J=\sum J_{\beta}, \beta \in I_{p} \subseteq I$, where $I_{p}=\left\{\alpha \in I \mid p^{\omega} J_{\alpha}=J_{\alpha}\right\}$. Clearly $p^{\omega} J$ is a direct summand of $J$.

Before we state the major theorem we prove

Proposition 2.2. Let $J=K \oplus L$ be a torsion-free group and let $T$ be a torsiongroup such that $\operatorname{Ext}(K, T)=0$. If $G \in \in \operatorname{Ext}(J, T)$ then $G=K \oplus G_{L}$ where $G_{L} \in \epsilon$ $\operatorname{Ext}(L, T)$.

Proof. Since $J=K \oplus L$ and $\operatorname{Ext}(K, T)=0, \operatorname{Ext}(L, T) \cong \operatorname{Ext}(J, T)$ under the map $\beta$ induced by the projection map $J \rightarrow L$. Hence every element of $\operatorname{Ext}(J, T)$ has the form

$$
\left(0 \rightarrow T \rightarrow G_{L} \rightarrow L \rightarrow 0\right)=\beta\left(0 \rightarrow T \rightarrow G_{L} \oplus K \rightarrow J \rightarrow 0\right) .
$$

Corollary 2.3. If every $G_{L} \in \in \operatorname{Ext}(L, T)$ is a direct sum of p-mixed groups then every $G \in \in \operatorname{Ext}(J, T)$ is also a direct sum of p-mixed groups.

THEOREM 2.4. Let $T=\sum T_{p}$ be a torsion group and $J=\sum J_{\alpha}, \alpha \in I$, be a completely decomposable group where $r\left(J_{\alpha}\right)=1$ for each $\alpha \in I$ and $\tau\left(J_{\alpha}\right)=\left(k_{1}^{\alpha}, k_{2}^{\alpha}, \ldots\right)$. Then the following two conditions are necessary and sufficient for every $G \in \in \operatorname{Ext}(J, T)$ to be a direct sum of p-mixed groups:

(A) If two primes $p \neq q$ are such that the orders of the elements in $T_{p} \mid p^{\omega} T_{p}$ and $T_{q} / q^{\omega} T_{q}$ are unbounded then $p^{\omega} J \cap q^{\omega} J=0$. 
(B) If $Q=\left\{p \mid p T_{p} \neq T_{p}\right\}$ is an infinite set of primes then $k_{i}^{\alpha}=0$ for all $\alpha \in I$ and for all but a finite number of $p_{i} \in Q$.

Before we prove the sufficiency of this theorem we will state Baer's Theorem $[1,8.1]$ in the case that $r(J)=1$. This states that $\operatorname{Ext}(J, T)=0$ if and only if (i) whenever the orders of the elements in $T_{p} / p^{\omega} T_{p}$ are unbounded then $p^{\omega} J=0$ and (ii) if $Q=\left\{p \mid p T_{p} \neq T_{p}\right\}$ is an infinite set of primes then $k_{i}=0$ for all but a finite number of the primes $p_{i} \in Q$ where $\tau(J)=\left(k_{1}, k_{2}, \ldots\right)$.

Proof of the sufficiency of Theorem 2.4. Let $P$ be the set of primes such that the orders of the elements in $T_{p} / p^{\omega} T_{p}$ are unbounded. Referring to our remarks on completely decomposable groups we have $p^{\omega} J=\sum J_{\beta}, \beta \in I_{p} \subseteq I$ where $J=\sum J_{\alpha}$, $\alpha \in I$. (A) tells us that $I_{0}=\bigcup I_{p}$ is a disjoint union so $\sum p^{\omega} J, p \in P$, is a direct sum. In fact if $K=\sum J_{\gamma}, \gamma \in I-I_{0}$ then $J=K \oplus \sum p^{\omega} J$.

Now $\operatorname{Ext}(K, T)=\prod \operatorname{Ext}\left(J_{y}, T\right)=0$ by Baer's Theorem. So, by Corollary 2.3, we need only show that every extension of $T$ by $\sum p^{\omega} J, p \in P$, is a direct sum of $p$-mixed groups.

Now $p^{\omega} J=\sum J_{\alpha}, \alpha \in I_{p}$. $(A)$ says that $q^{\omega} J_{\alpha}=0$ for $\alpha \in I_{p}$ and for $q \neq p$. Since each of these $J_{\alpha}$ satisfies $(B)$ for the set $Q-\{p\}$ we get $\operatorname{Ext}\left(J_{\alpha}, T^{p}\right)=0$. So $\operatorname{Ext}\left(p^{\omega} J, T^{p}\right)$ $=\prod_{\alpha} \operatorname{Ext}\left(J_{\alpha}, T^{p}\right)=0$. But this means that $\sum p^{\omega} J$ satisfies the conditions of Theorem 2.1 and so every $G \in \in \operatorname{Ext}\left(\sum p^{\omega} J, T\right)$ is a direct sum of $p$-mixed groups.

3. Necessity of $(A)$. If the $p$-primary component $T_{p}$ of $T$ has the property that $T_{p} / p^{\omega} T_{p}$ is a group of bounded order then $T_{p}=D \oplus C$ where $D$ is divisible and $C$ is a pure subgroup of bounded order. Hence $T_{p}$ will be a direct summand of any group $G$ containing $T$ and we need not concern ourselves with these primary components.

The proof of the following lemma is in Baer [1].

LEMMA 3.1. Let $G$ be a mixed group and $J$ a torsion-free group with $G / t G \subseteq J$. Then there exists a mixed group $G^{\prime}$ satisfying:

(i) $G \subseteq G^{\prime}$ and $t G=t G^{\prime}$.

(ii) $G^{\prime} \mid t G^{\prime}=J$ (i.e., there is an isomorphism between $G^{\prime} \mid t G^{\prime}$ and $J$ leaving invariant the elements of $G / t G)$.

Proposition 3.2. Let $J$ be a completely decomposable group such that $q^{\omega} J=J$ for some prime $q$ and let $T_{q}$ be a q-primary group such that the elements of $T_{q} / q^{\omega} T_{q}$ have unbounded orders. Then there is an extension

$$
0 \rightarrow T_{q} \rightarrow S \rightarrow J \rightarrow 0
$$

such that $T_{q}$ is not a direct summand of the complete inverse image in $S$ of any nonzero pure subgroup of $J$ (in particular $T_{q}$ is not a direct summand of $S$ ).

Proof. Let $J=\sum J_{\alpha}, \alpha \in I$ be a decomposition of $J$ into groups of rank one. Because $J=q^{\omega} J$ we can find, for a given $j_{\alpha} \in J_{\alpha}$ and for each $\alpha \in I$, elements $\bar{f}_{\alpha}^{i}$ in $J_{\alpha}$ for $i=0,1,2, \ldots$ with

$$
\bar{f}_{\alpha}^{0}=j_{\alpha} \quad \text { and } \quad q \bar{f}_{\alpha}^{i}=\bar{f}_{\alpha}^{i-1}
$$


Also (see page 203 of [2]) there are elements $c_{i}, d_{i} \in T_{q}$ for $i=1,2, \ldots$ satisfying the equations $d_{i}=\sum_{s=0}^{i=1} q^{s} c_{s+1}$ with the property that the orders of the elements $d_{i}$ tend to infinity with $i$ and that they are elements of lowest order in their cosets $\bmod q^{i} T_{q}$.

Now form the mixed group $K$ generated by the elements of $T_{q}$ along with the elements $\left\{f_{\alpha}^{i} \mid \alpha \in I, i=0,1,2, \ldots\right\}$ and which has all the relations of $T$, commutativity relations and the additional relations $q f_{\alpha}^{i}-f_{\alpha}^{i-1}=c_{i}$ for $i>0$. Then $K$ is a mixed group with $t K=T_{q}$ and $K / t K$ isomorphic to a subgroup of $J$ where $f_{\alpha}^{i} \rightarrow \bar{f}_{\alpha}^{i}$. By Lemma 3.1 we can find a mixed group $S$ such that $t S=t K=T_{q}, K \subseteq S$ and $S$ $\in \in \operatorname{Ext}\left(J, T_{q}\right)$ where the epimorphism $\pi: S \rightarrow J$ with kernel $T_{q}$ also sends $f_{\alpha}^{i} \rightarrow f_{\alpha}^{i}$.

Now let $x$ be a nonzero element of any pure subgroup of $J$ and suppose $T_{q}$ is a direct summand of the complete inverse image of this pure subgroup under $\pi$. Then $T_{q}$ will also be a direct summand of $\pi^{-1}(C(x))$ where $C(x)$ is the minimal pure subgroup of $J$ containing $x$. Write $\pi^{-1}(C(x))=T_{q} \oplus H$. We shall show that this assumption gives rise to a contradiction.

Since $x \in J=\sum J_{\alpha}, \alpha \in I$, we can write $x=x_{\alpha(1)}+\cdots+x_{\alpha(m)}$ uniquely where $0 \neq x_{\alpha(i)} \in J_{\alpha(i)}$ for $1 \leqq i \leqq m$. Since $r(C(x))=1, C(x)$ is contained in $\sum_{i=1}^{m} J_{\alpha(i)}$. Now the $m$ independent elements, $\widehat{f}_{\alpha(i)}^{0} \in J_{\alpha(i)}$, which we chose above, must form, along with $x$, a dependent set in $\sum_{i=1}^{m} J_{\alpha(i)}$. So we can find integers $n, n_{1}, \ldots, n_{m}$ such that

$$
n x=n_{1} \bar{f}_{\alpha(1)}^{0}+\cdots+n_{m} \bar{f}_{\alpha(m)}^{0} .
$$

Now, for each $i=0,1,2, \ldots$, we have

$$
q^{i}\left(\sum n_{j} \bar{f}_{\alpha(j)}^{i}\right)=\sum n_{j} \bar{f}_{\alpha(j)}^{0} \in C(x)
$$

where the sums are taken over $j=1,2, \ldots, m$. But $C(x)$ is a pure subgroup of the torsion-free group $J$ so we must have $\sum n_{j} \bar{f}_{\alpha(j)}^{i} \in C(x)$ for all $i=0,1,2, \ldots$ Thus the elements $\sum n_{j} f_{\alpha(j)}^{i} \in \pi^{-1}(C(x))=H \oplus T_{q}$. Therefore, for each $i$, we get the unique expression

$$
\sum_{j=1}^{m} n_{j} f_{\alpha(j)}^{i}=h^{i}+t_{q}^{i}
$$

where $h^{i} \in H$ and $t_{q}^{i} \in T_{q}$. Now we apply the relations in (1) and get

$$
q\left(\sum n_{j} f_{\alpha(j)}^{i}\right)=\sum n_{j} f_{\alpha(j)}^{i-1}+c_{i} \sum n_{j} .
$$

We will let $\sum n_{j}=N$. Multiplying by $q$ in (2) and using (3) gives

$$
\sum n_{j} f_{\alpha(j)}^{i-1}=q h^{i}+\left(q t_{q}^{i}+N c_{i}\right) .
$$

Since $q t_{q}^{i}+N c_{i}$ is an element of $T_{q}$ we must have, because of the uniqueness of the expression (2) that

$$
t_{q}^{i-1}=q t_{q}^{i}-N c_{i}
$$

Therefore

$$
q^{i} t_{q}^{i}-t_{q}^{0}=N \sum_{s=0}^{i-1} q^{s} c_{s+1}=N d_{i}
$$


But then $-t_{q}^{0} \equiv N d_{i} \bmod q^{i} T_{q}$ for all $i$. Since the $d_{i}$ were chosen having the property that $O\left(d_{i}\right)$ tend to infinity with $i$ then the same is true for $O\left(N d_{i}\right)$. To produce our contradiction we want to show that $O\left(-t_{q}^{0}\right) \geqq O\left(N d_{i}\right)$ for infinitely many $i$.

We can assume $N=q^{k}$ and so we choose $i_{0}$ such that $O\left(d_{i}\right)>q^{k}$ for $i \geqq i_{0}$. Thus $q^{k}<O\left(d_{i}\right) \leqq O\left(d_{i}+q^{i} t_{q}\right)$ for every $t_{q} \in T_{q}$ by the way the $d_{i}$ were chosen. So

$$
O\left(q^{k} d_{i}\right) \leqq O\left(q^{k}\left(d_{i}+q^{i} t_{q}\right)\right) \text {. }
$$

So for each $i \geqq i_{0}$ we need only show that $-t_{q}^{0}=q^{k}\left(d_{i}+q^{i} t_{q}\right)$ for some $t_{q} \in T_{q}$. By successive applications of (4) we get $t_{q}^{i}=q^{k} t_{q}^{i+k}+q^{k} t_{q}^{\prime}$ for some $t_{q}^{\prime} \in T_{q}$. Therefore $t_{q}^{i}=q^{k} t_{q}^{\prime \prime}$ for $t_{q}^{\prime \prime} \in T_{q}$. Hence by (5)

$$
-t_{q}^{0}=q^{k} d_{i}+q^{i} t_{q}^{i}=q^{k}\left(d_{i}+q^{i} t_{q}^{\prime \prime}\right) .
$$

This completes the proof.

We can now complete the proof of the necessity of $(A)$. Let $T$ be a torsion group such that for some two primes $p \neq q$ the elements of $T_{p} / p^{\omega} T_{p}$ and of $T_{q} / q^{\omega} T_{q}$ have unbounded order; and let $J$ be a completely decomposable group such that $\left(p^{\omega} J\right) \cap\left(q^{\omega} J\right) \neq 0$. Then, since $J$ is a direct sum of groups of rank 1 ,

for some $K$. Let

$$
J=\left(p^{\omega} J \cap q^{\omega} J\right) \oplus K
$$

(1) $0 \longrightarrow T=T_{p} \oplus T_{q}^{\prime} \oplus T^{\prime} \stackrel{i}{\longrightarrow} S \oplus K \oplus T^{\prime} \stackrel{v}{\longrightarrow} J=\left(p^{\omega} J \cap q^{\omega} J\right) \oplus K \longrightarrow 0$ be any element of $\operatorname{Ext}(J, T)$ for which $i$ is the identity on $T^{\prime}$ and $\nu$ is the identity on $K$ and $\nu(S)=p^{\omega} J \cap q^{\omega} J$. In any decomposition of $S \oplus K \oplus T^{\prime}$ into a direct sum of groups whose torsion subgroups are the primary components of $T$, the primary components of $T^{\prime}$ will be direct summands of the terms in which they occur. Thus it will be sufficient to construct our example (1) for the case $T^{\prime}=0$ (and later add $T^{\prime}$ ).

To further reduce our construction to the case $K=0$ it will be sufficient to show that any direct sum decomposition $S \oplus K=G_{p} \oplus G_{q}$ with $t\left(G_{p}\right)=T_{p}$ and $t\left(G_{q}\right)=T_{q}$ induces a direct sum decomposition $S=\left(S \cap G_{p}\right) \oplus\left(S \cap G_{q}\right)$. But this follows from the fact that $S$ is the set of elements $x$ of $S \oplus K$ for which $v(x) \in p^{\omega} J \cap q^{\omega} J$.

Now construct two extensions which fill the bill of Proposition 3.2 (the first with $p$ in place of $q$ ):

$$
\begin{aligned}
& 0 \longrightarrow T_{p} \longrightarrow S_{1} \stackrel{v_{1}}{\longrightarrow} J \longrightarrow 0 \\
& 0 \longrightarrow T_{q} \longrightarrow S_{2} \stackrel{v_{2}}{\longrightarrow} J \longrightarrow 0
\end{aligned}
$$

and let $S=\left\{\left(s_{1}, s_{2}\right): \nu_{1} s_{1}=\nu_{2} s_{2}\right\}$. We can identify $T_{p}$ with the subgroup $\left(T_{p}, 0\right)$ of $S$ and $T_{q}$ with $\left(0, T_{q}\right) . T=T_{p} \oplus T_{q}$ is then the torsion subgroup of $S$ and we can construct the following commutative diagram

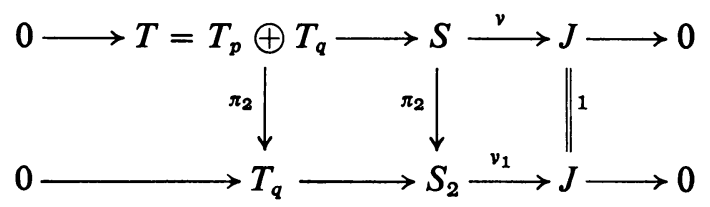


where $v\left(s_{1}, s_{2}\right)=v_{1}\left(s_{1}\right)=v_{2}\left(s_{2}\right)$ and $\pi_{2}$ is the coordinate projection map. Suppose now, by way of contradiction, that $S=G_{p} \oplus G_{q}$ with the torsion subgroups of $G_{p}$ and $G_{q}$ equal to $T_{p}$ and $T_{q}$ respectively. Then $J=\nu(S)=\nu\left(G_{p}\right) \oplus \nu\left(G_{q}\right)$ with at least one term, say the first, nonzero. The complete inverse image of $\nu\left(G_{p}\right)$ in $S$ is $G_{p} \oplus T_{q}$. Thus by (2) the complete inverse image (via $\left.\nu_{1}\right)$ of $\nu\left(G_{p}\right)$ in $S_{2}$ is $\pi_{2}\left(G_{p}\right) \oplus T_{q}$, the sum being direct since ker $\pi_{2}=T_{p}$. This shows that $\pi_{2}\left(G_{p}\right)$ is torsion-free. But this contradicts Proposition 3.2, and hence completes the proof of the necessity of $(A)$.

4. The necessity of $(B)$. Let $T$ be a torsion group such that the set of primes $Q=\left\{p \mid p T_{p} \neq T_{p}\right\}$ is infinite. Take a torsion-free group of rank one, $J$, with $\tau(J)=\left(k_{1}, k_{2}, \ldots\right)$ where $k_{i}>0$ for an infinite number of $i$ with $p_{i} \in Q$. We then construct an extension of $T$ by $J$ which is not a direct sum of $p$-mixed groups. With this accomplished we will then extend to the case where $J$ is completely decomposable.

With this $J$ and $T$ choose $j^{\prime} \in J, j^{\prime} \neq 0$. Since $\tau\left(j^{\prime}\right)=\tau(J)$ we have $H_{p}\left(j^{\prime}\right)>0$ for an infinite number of $p \in Q$. Let $W=\left\{p \in Q \mid H_{p}\left(j^{\prime}\right)>0\right\}$.

So for each $p \in W$ choose $j_{p}^{\prime} \in J$ such that $p j_{p}^{\prime}=j^{\prime}$. Form the mixed group $K$ generated by the elements of $T$ and by elements $j$ and $j_{p}$ for $p \in W$, demanding that $K$ has all the relations of $T$, all commutative relations along with

$$
p j_{p}-j=t_{p} \quad \text { for each } p \in W, \quad t_{p} \in T_{p}
$$

where $t_{p}$ is chosen so that $t_{p} \notin p T_{p}$ (which is possible since $p T_{p} \neq T_{p}$ for $p \in Q$ and thus for $p \in W$ ). We now have $t K=T$ and $K / t K$ isomorphic to a subgroup of $J$ where $j \rightarrow j^{\prime}$ and $j_{p} \rightarrow j_{p}^{\prime}$.

What we intend to show is that $K$ can have no direct summand $S$ which is a torsion subgroup of $K$ containing all but a finite number of the primary components of $T$. We do this by contradiction.

So let $K=S \oplus H$ where $S \subseteq T$ and contains all but a finite number of the primary components of $T$. Let the subset $V \subseteq W$ be defined by $V=W=\left\{p \mid t_{p} H \neq 0\right\}$. Since $H$ can have only a finite number of nonzero primary components it follows that $V$ is an infinite subset of $Q$.

Now since $K=S \oplus H$ is a direct sum we can write

$$
j=s+h \quad \text { and } \quad j_{p}=s^{p}+h^{p}
$$

uniquely where $s, s^{p} \in S$ and $h, h^{p} \in H$. Also, for $p \in V$, we have

$$
p h^{p}-h=p\left(j_{p}-s^{p}\right)-(j-s)=t_{p}-p s^{p}+s .
$$

But $p h^{p}-h \in H$ and $t_{p}-p s^{p}+s \in S$ (since $T_{p} \subseteq S$ for $p \in V$ ) so $p h^{p}-h=t_{p}-p s^{p}+s=0$.

Now the torsion element $s$, when written in its unique fashion as a sum of elements of the primary components of $T$, can have only a finite number of nonzero components. So we let $V^{\prime}$ be that subset of $V$ for which $s$ has zero $p$-primary components. $V^{\prime}$ is still an infinite subset of $Q$. Most important is the fact that $V^{\prime}$ is nonempty! 
We now read the equations $t_{p}-p s^{p}+s=0$ in $T_{p}$ for $p \in V^{\prime}$, that is we look at the $p$-primary component of $t_{p}-p s^{p}+s$ for $p \in V^{\prime}$. If we let the $p$-primary component of $s^{p}$ be $s_{p}^{p}$ we get $t_{p}-p s_{p}^{p}=0$ (since the $p$-primary component of $s$ is zero for $\left.p \in V^{\prime}\right)$. But $t_{p}-p s_{p}^{p}=0$ gives us $p s_{p}^{p}=t_{p}$, in other words $t_{p} \in p T_{p}$ which is contrary to the way $t_{p}$ was chosen. Hence $K$ can have no decomposition such as $K=S \oplus H$ where $S$ has the properties mentioned.

We can now, by applying Lemma 3.1, find a mixed group $G$ satisfying:

(i) $K \subseteq G$,

(ii) $t K=t G=T$,

(iii) $G / t G=J$ (leaving $K / t G$ invariant).

If $G$ had the above direct sum decomposition, namely $G=S \oplus H^{\prime}$ where $S \subseteq T$ and $S$ contains all but a finite number of the primary components of $T$ then it would happen, since $T \subseteq K$ and $S \subseteq T$, that

$$
K=K \cap G=(K \cap S) \oplus\left(K \cap H^{\prime}\right)=S \oplus\left(K \cap H^{\prime}\right)
$$

which we have shown to be impossible. So we have proven

THEOREM 4.1. Let $T=\sum T_{p}$ be a torsion group such that the set $Q=\left\{p \mid p T_{p} \neq T_{p}\right\}$ is infinite. Let $J$ be a torsion-free group of rank one with type $\tau(J)=\left(k_{1}, k_{2}, \ldots\right)$ where $k_{i}>0$ for an infinite number of the $p_{i} \in Q$. Then there is a mixed group $G \in \in \operatorname{Ext}(J, T)$ having no decomposition of the form $S \oplus H$ where $S$ is torsion and contains an infinite number of the $T_{p}$ with $p \in Q$.

COROllary 4.2. The group $G$ of Theorem 4.1 is not a direct sum of p-mixed groups.

Proof. This follows because a rank one group $J$ is indecomposable.

We now have the necessary ammunition to complete the proof of Theorem 2.4 by showing that $(\boldsymbol{B})$ is necessary. We have $J=\sum J_{\alpha}$ where $r\left(J_{\alpha}\right)=1$ for each $\alpha \in I$. If it happened that for some $\beta \in I, \tau\left(J_{\beta}\right)=\left(k_{1}^{\beta}, k_{2}^{\beta}, \ldots\right)$ and $k_{i}^{\beta}>0$ for an infinite number of the $p_{i} \in Q$ then we can get $G \in \in \operatorname{Ext}\left(J_{\beta}, T\right)$ satisfying Theorem 4.1. Let $K=G \oplus \sum J_{\alpha}, \alpha \in I, \alpha \neq \beta$. Then $K \in \in \operatorname{Ext}(J, T)$.

Suppose now that $K$ is a direct sum of $p$-mixed groups, say $K=\sum K_{p}$. Since $T \subseteq G, G / T$ is a subgroup of the factor group $K / T=\sum K_{p}^{\prime}$ where $K_{p}^{\prime} \cong K_{p} / T_{p}$. Furthermore $G / T$ is a group of rank one (it is isomorphic to $J_{\beta}$ ) so $G / T$ " hits" only a finite number of the $K_{p}^{\prime}$ 's, say $G / T \subseteq K_{p(1)}^{\prime}+\cdots+K_{p(n)}^{\prime}$. Let $\pi_{K}: K \rightarrow K / T$ be the natural quotient map. Then $G=\pi_{K}^{-1}(G / T)$ so

Moreover

$$
G \subseteq \pi_{K}^{-1}\left(K_{p(1)}^{\prime}+\cdots+K_{p(n)}^{\prime}\right) \text {. }
$$

$$
\pi_{K}^{-1}\left(K_{p(1)}^{\prime}+\cdots+K_{p(n)}^{\prime}\right)=K_{p(1)} \oplus \cdots \oplus K_{p(n)} \oplus S
$$

where $S \subseteq T$ and $S=\sum T_{q}, q \neq p(i)$ for any $i, 1 \leqq i \leqq n$. Since $T \subseteq G$ this implies $S$ is a direct summand of $G$ which contradicts our choice of $G$ in accordance with Theorem 4.1. 


\section{BIBLIOGRAPHY}

1. R. Baer, The subgroup of the elements of finite order of an Abelian group, Ann. of Math. 37 (1936), 766-781.

2. A. G. Kurosh, The theory of groups, Vol. 1, 2nd English edition, Chelsea, New York, 1952.

UNIVERSTTY OF NOTRE DAME,

NOTRE DAME, INDIANA

UNIVERSTTY OF VIRomia,

Charlottesville, Viroinia 